\title{
Infraestructura y calidad educativa entre los pueblos indígenas originarios sonorenses
}

Infrastructure and Quality of Education among Indigenous Peoples from Sonora

Infrastructure et qualité éducative parmi les peuples indigènes originaires sonorenses

Infraestrutura e qualidade educativa entre os povos indígenas originários de Sonora

Fecha de recepción: 21 DE FEBRERO DE 2014/Fecha de aceptación: 9 DE NOVIEMBRE DE 2015/Fecha de disponibilidad en línea: 15 DE MARZO DE 2016

Encuentre este artículo en http://magisinvestigacioneducacion.javeriana.edu.co/

Escrito por José Ángel Vera-Noriega Centro de Investigación en Alimentación y Desarrollo A. C., CIAD SONORA, MÉXICO avera@ciad.mx

Diana Luque-Agraz Centro de Investigación en Alimentación y Desarrollo A. C., CIAD

SONORA, MÉxICO dluque@ciad.mx

Gildardo Bautista-Hernández Centro de Investigación en Alimentación y Desarrollo A. C., CIAD

\section{Resumen}

El objetivo del artículo se centra en el análisis de la percepSONORA, MÉXICO ción entre los pueblos indígenas originarios de Sonora sogilbher@gmail.com bre la infraestructura y calidad educativa para la formación de sus descendientes. Participaron 1.329 personas con un promedio de 50 años de edad y desviación estándar de 16 años, 928 mujeres y 401 hombres. Los resultados indican la prevalencia de una percepción de falta de infraestructura para mejorar la calidad educativa de los niños y jóvenes indígenas. Además, se encontraron diferencias entre los siete pueblos indígenas con respecto a la problemática educativa. Los grupos que mejor están ubicados, en cuanto a acceso educativo, son aquellos que exhiben mayor desplazamiento de su lengua materna.

\section{Palabras clave}

Población indígena; calidad de la educación; educación intercultural; México

\begin{abstract}
Transferencia a la práctica
Se requiere un conocimiento profundo de las características y necesidades de los siete pueblos indígenas originarios de Sonora y de cómo alcanzar el ejercicio pleno de su derecho a la educación. Las evidencias señalan una problemática generalizada en este sector social, no obstante con diferentes matices. Estos hallazgos son fundamentales para reforzar las políticas de interculturalidad e inclusión que integren la diversidad cultural y lingüística, así como las aspiraciones que caracterizan a cada pueblo, sobre todo, a las nuevas generaciones que, en ocasiones, difieren de sus autoridades tradicionales.
\end{abstract}

Para citar este artículo / To cite this article / Pour citer cet article / Para citar este artigo

Vera-Noriega, J. Á.; Luque-Agraz, D. \& Bautista-Hernández, G. (2016). Infraestructura y calidad educativa entre los pueblos indígenas originarios sonorenses. magis, Revista Internacional de Investigación en Educación, 8 (17), 47-64. http://dx.doi.org/10.11144/Javeriana.m8-17.icep 


\section{Keywords}

Indigenous population; quality of education; intercultural education; Mexico

\section{Mots clés}

Peuples indigènes; qualité de l'éducation; éducation interculturel; Mexique

\section{Abstract}

This article analyzes the perception of indigenous peoples from Sonora regarding infrastructure and quality of education for the instruction of their descendants. This research involved 1,329 people, with an average age of 50 and standard deviation of 16 years, from which 928 were women and 401 were men. Results indicate the prevalence of a perceived lack of infrastructure to improve quality of education for indigenous children and youth. In addition, we found differences between the seven indigenous peoples with regard to educational problems. Groups that are better placed, in terms of access to education, are those that exhibit larger displacement of their native language.

\section{Transfer to practice}

A deep understanding of the characteristics and needs of the seven indigenous peoples of Sonora and understanding how to achieve the full exercise of their right to education is required. Evidence indicates a problem that has spread in this social group, although with different nuances. These findings are essential to strengthen the policies of interculturality and inclusion that integrate cultural and linguistic diversity, as well as the aspirations that characterize each one of this groups, especially the younger generations, which sometimes differ from traditional authorities.

\section{Résumé}

L'objectif de l'article se trouve dans l'analyse de la perception parmi les peuples indigènes originaires de Sonora par rapport à l'infrastructure et qualité éducative pour la formation de leur descendantes. Dans l'étude 1. 329 personnes ont participés avec une moyenne d'âge de 50 ans et déviation standard de 16 ans, 928 femmes et 401 hommes. Les résultats indiquent la prévalence d'une perception de manque d'infrastructure pour améliorer la qualité éducative des enfants et jeunes indigènes. D'ailleurs, on a trouvé de différences entre les sept peuples indigènes à l'égard de la problématique éducative. Les groupes qu sont mieux installés, par rapport à l'accès éducatif sont ceux qui montrent un écart plus grand de sa langue maternelle.

\section{Transfert à la pratique}

On requiert d'une connaissance plus profonde des caractéristiques et besoins de sept peuples indigènes originaires de Sonora et de comment parvenir à l'exercice plein de leur droit à l'éducation. Les évidences montrent une problématique généralisée dans ce secteur social, néanmoins avec des différentes nuances. Ces recherches sont fondamentales pour renforcer les politiques d'interculturalité et d'inclusion qui intègrent la diversité culturelle et linguistique, ainsi que les aspirations qui caractérisent à chaque peuple, sur tout aux nouvelles générations qu ont parfois de différences par rapport aux autorités traditionnelles.

\section{Palavras-chave}

População indígena; qualidade da educação; educação intercultural; México

\section{Resumo}

O objetivo do artigo é analisar a percepção entre os povos indígenas originários de Sonora sobre a infraestrutura e qualidade educativa para a formação de seus descendentes. Participaram 1.329 pessoas com uma idade média de 50 anos e desvio padrão de 16 anos, sendo 928 mulheres e 401 homens. Os resultados indicam a prevalência de uma percepção de falta de infraestrutura para melhorar a qualidade educativa das crianças e jovens indígenas. Além do mais, encontraram-se diferenças entre os sete povos indígenas a respeito da problemática educativa. Os grupos que melhor estão localizados, quanto a acesso educativo, são aqueles que exibem maior deslocação de sua língua materna.

\section{Transferência à prática}

É necessário um conhecimento profundo das características e necessidades dos sete povos indígenas originários de Sonora e de como atingir o exercício pleno de seu direito à educação. As evidências assinalam uma problemática generalizada neste setor social, apresar de diferentes matizes. Estes resultados são fundamentais para reforçar as políticas de interculturalidade e inclusão que integrem a diversidade cultural e linguística, bem como as aspirações que caracterizam cada povo, sobretudo, as novas gerações que, em algumas ocasiões, diferem de suas autoridades tradicionais. 


\section{Introducción}

México es un país que se reconoce en su Constitución Política como una nación pluricultural sustentada en sus pueblos originarios (México, 1917, CP, Art. 2) y que dada su cultura, historia, lengua y usos y costumbres le dan sentido de pertenencia e identidad a la Nación (Comisión Nacional para el Desarrollo de los Pueblos Indígenas, CDI, 2014).

Entre los criterios para la cuantificación o visibilización de los pueblos indígenas', el II Conteo de Población y Vivienda del Instituto Nacional de Estadística y Geografía, INEGI, en 2005, utilizó el criterio de los hablantes de lengua indígena $(\mathrm{HLI})^{2}$, el cual invisibilizaba casi a la mitad de la población indígena. Para el censo de 2010, ya se aplicó el criterio de hogares con población indígena $(\mathrm{HPI})^{3}$, que se acercó a los criterios de autoadscripción utilizado por las comunidades indígenas (Luque, Martínez, Búrquez, Gómez, Nava \& Rivera, 2012c).

Siguiendo el criterio de INEGI de hogares con población indígena, en el cual el jefe y/o cónyuge habla una lengua indígena, la Comisión Nacional de Población (CONAPO, 2012) menciona que México tiene una población indígena de 14.252.243 personas, de estas, 7.114 .803 son hombres y 7.137.440 son mujeres.

El reconocimiento y las contribuciones a la riqueza multicultural que realizan los pueblos originarios de México contrastan con sus condiciones de rezago sistémico en prácticamente todos los temas de desarrollo, como salud, justicia y educación. La población indígena ha sido la última en contar con servicios públicos como agua potable, luz, carreteras cerca de sus comunidades, acceso al servicio de salud y educación (Schmelkes, 2010). Asimismo, concentra una problemática de alta marginación, pobreza multidimensional y bajos índices de desarrollo humano (CDI, 2009; CDI, 2014; CDI-PNUD, 2006).

El panorama educativo de este sector social exhibe los rezagos más profundos del país, ya que según el Instituto Nacional para la Evaluación de la Educación, INEE (2013), del total de esta población hablante de lengua indígena de 15 años o más, el $26,8 \%$ es analfabeta; se estima que en esta población, el $34,5 \%$ de las mujeres y el $19,6 \%$ de los hombres son analfabetas; mientras que el promedio de escolaridad es de quinto de primaria, ya que los niños indígenas a los 12 años de edad — al haber concluido la primaria - abandonan los estudios (CDI-PNUD, 2010).

La Encuesta Nacional de la Dinámica Demográfica, ENADID, en materia educativa, el 91,0\% de la población indígena menor de 15 años asiste a la escuela. Asimismo, 4,3\% de la población joven indígena no cuenta con ningún nivel de escolaridad, $11,7 \%$ tiene al menos un grado de primaria, $21,3 \%$ terminó la primaria y $62,6 \%$ concluyó la secundaria (Comisión Nacional de Población, CONAPO, 2010), pero solo dos de cada 100 integrantes de los grupos étnicos llegan a la educación superior (Avilés, 2011).

En la trayectoria escolar, los estudiantes indígenas se enfrentan a problemas de acceso, deserción y eficiencia terminal. Estas problemáticas están asociadas a una deficiente infraestructura educativa, a las aspiraciones de los padres y las limitaciones económicas. Asimismo, los

1 Se utiliza el término "pueblos indígenas" siguiendo las convenciones internacionales para designar aquellas colectividades que se autoidentifican como una comunidad originaria en el contexto de los estados nacionales (ONU, 2007; CIDH; Convenio 169).

2 Persona de 5 años y más que habla alguna lengua indígena.

3 Población que vive en hogares donde el jefe/a, cónyuge o ascendentes hablan lengua indígena.
Descripción del artículo | Article description | Description de l'article | Artigo descrição

Este artículo de investigación es producto del grupo de investigación interdisciplinaria conformada por los autores, que pertenecen al Centro de Investigación en Alimentación y Desarrollo (CIAD). Se presentan los resultados vinculados con el estado de la infraestructura y la calidad educativa en los siete pueblos originarios de Sonora. 
jóvenes de los pueblos originarios se ven obligados a trabajar a una edad temprana, ya sea en el lugar de origen o a emigrar hacia zonas de mayor desarrollo (Castillo, 2005).

Otro factor que influye en el interés por concluir estudios de educación media y superior es qué oportunidades laborales tendrán, ya que consideran que a pesar de invertir en sus estudios, lo más probable es que terminen realizando labores de mantenimiento en centros de trabajo, que estén desempleados, o incluso se vean obligados a migrar como indocumentados a otro país.

Para garantizar el pleno derecho a la educación de esta población, en las últimas décadas, el Estado mexicano ha desarrollado diversos prototipos didácticos; sin embargo, todavía prevalece el rezago educativo y social en las diversas regiones indígenas de México (Muñoz, 2001). La educación bilingüe intercultural se promueve como una opción para mejorar la calidad educativa y contrarrestar los altos índices de reprobación y deserción escolar (Secretaría de Educación Pública, SEP, 2011).

\section{Marco referencial Educación intercultural e inclusiva}

Frente a la diversidad cultural y las condiciones educativas de los pueblos indígenas surge la propuesta intercultural, como una aspiración en el marco de la redefinición de México como país pluricultural y plantea una relación horizontal entre las culturas. Entre los grupos culturales, debe haber vínculos fundados en el respeto desde planos de igualdad y democracia. Es decir, no admitir asimetrías o desigualdades entre culturas mediadas por el poder, que benefician a un grupo cultural por encima de otro u otros (Schmelkes, 2013a).

Uno de los retos de la educación intercultural es el de superar las constantes fricciones entre el Estado nacional y el carácter pluricultural de México. Por ejemplo, la nación exige la unificación ideológica y cultural para lograr "una" identidad cultural, teniendo la educación pública como una de sus principales plataformas de acción ("una" historia, "una" lengua). Mientras, los pueblos indígenas siguen luchando por conservar su autonomía política, la cual se funda en la cultura tradicional, la lengua materna y el territorio 4 .

Entre los años 2005 y 2010, en México, la población hablante de alguna lengua indígena disminuyó

4 En 2012, a raíz de la reforma educativa, en el municipio de Cherán, estado de Michoacán, la población purépecha se negó a aprender inglés y computación como temas prioritarios por encima del aprendizaje de su lengua materna. En los enfrentamientos, ocho comuneros resultaron muertos. de 34\% a 27\%. Según el Índice de Reemplazo Etnolingüístico (CDI, 2009), el desplazamiento de la lengua materna es un fenómeno generalizado en prácticamente todos los pueblos indígenas contemporáneos. En Sonora, la lengua de los mayos exhibe un proceso acelerado de desaparición (Moctezuma, 2001), mientras que las lenguas cucapá y pápago están prácticamente ya extintas, y las de los yaquis, seris, pimas y guarijíos están en riesgo.

Prevalece una clara política educativa discriminatoria, intrínseca en el sistema que desconoce los conocimientos locales, como el manejo de la biodiversidad, que son transmitidos generalmente, de manera oral, por medio de prácticas de subsistencia, que en su conjunto, se consideran el "patrimonio biocultural" de los pueblos (Boege, 2008; Nabhan, 2003; Toledo \& Barrera, 2008).

En el caso de Sonora, los pueblos con mayor nivel educativo presentan un menor índice de conservación de los recursos naturales de su territorio (Luque, Martínez, Búrquez, Gómez, Nava \& Rivera, 2012b). Por esto, frente a la crisis global ambiental, climática e hídrica, la educación biocultural está adquiriendo pertinencia en el seno de las comunidades indígenas.

La educación bilingüe intercultural que se promueve como una opción para mejorar las condiciones educativas de los pueblos originarios (SEP, 2011), no ha logrado garantizar el derecho constitucional a toda la población indígena. Y tampoco ha sido capaz de ser una plataforma para la defensa de los derechos reconocidos en la Declaración de las Naciones Unidas sobre los Derechos de los Pueblos Indígenas (ONU, 2007).

En este sentido, el Estado mexicano debe caminar hacia el reconocimiento, promoción, y vigencia de los Derechos de los Pueblos Indígenas, lo que implica hacer efectivos los Derechos no solo a la educación, sino también a la salud, la alimentación, la vivienda, el trabajo y a un desarrollo pleno de sus capacidades, siempre desde un marco de equidad e interculturalidad (CDI, 2014).

Un sistema educativo intercultural e inclusivo que busca contribuir a superar las desigualdades y reducir la brecha social, debe realizar mayores esfuerzos para que las escuelas se adapten a las necesidades y demandas de los alumnos (Blanco, 2006; Schmelkes, 2013b), y el establecimiento progresivo de vínculos dialógicos para entenderse, de puentes y veredas alternativas de comunidades humanas, de reconocimiento mutuo y un proyecto en común (Almeida-Acosta, 2013).

\section{Población indígena de Sonora y procesos educativos}

Hay siete pueblos indígenas que se consideran originarios de Sonora (cucapá, seri, yaqui, mayo, pápago, 
pima y guarijío, que representan el 78,5\% de la población total indígena de Sonora). Estos son prehispánicos y aún habitan sus territorios ancestrales, y sobre todo, su organización sociopolítica comunitaria que los identifica como pueblos sigue vigente. Los demás son grupos de indígenas migrantes, generalmente, jornaleros agrícolas provenientes de diferentes Estados del país (tabla 1).

En las comunidades indígenas de Sonora, prevalecen los criterios de autoadscripción y reconocimiento comunitario, los cuales indican que deben ser los sujetos indígenas quienes se identifiquen como tales. Sin embargo, no es suficiente que alguien se identifique de manera personal como indígena, este individuo tiene que ser reconocido por los miembros del grupo en cuestión (Luque, Martínez, Búrquez, Gómez, Nava \& Rivera, 2012b). Así, en el Estado de Sonora, el Instituto Nacional de Estadística y Geografía, INEGI (2005), registró 95.507 pobladores indígenas, cifra que contrasta con las 137.560 personas (registradas por censos comunitarios), distribuidas en alrededor de 50 grupos etnolingüísticos (Luque, Martínez, Búrquez, Gómez, Nava \& Rivera, 2012b).

Tabla 1

Población indígena de Sonora, su grupo lingüístico y su territorio

\begin{tabular}{l|l|c|c|c}
\hline $\begin{array}{c}\text { Autodenominación - } \\
\text { Nombre común }\end{array}$ & \multicolumn{1}{|c|}{$\begin{array}{c}\text { Grupo } \\
\text { lingüístico }\end{array}$} & HLI* & HPI** & $\begin{array}{c}\text { Conteo } \\
\text { Comunitario*** }\end{array}$ \\
\hline Yoreme-Mayo & Yuto-Azteca & 34.673 & 58.892 & 65.000 \\
\hline Yoreme-Yaqui & Yuto-Azteca & 15.903 & 21.789 & 36.409 \\
\hline Mahkurawe-Guarijío & Yuto-Azteca & 1.365 & 1.414 & 1.580 \\
\hline O'ob-Pima bajo & Yuto-Azteca & 488 & 658 & 971 \\
\hline Tohono Otam-Pápagos & Yuto-Azteca & 318 & 253 & 2.000 \\
\hline Es-pei-Cucapá & Yumana & 266 & 70 & 470 \\
\hline Comcáac- Seri & Hokana & 735 & 756 & 1.050 \\
\hline Kikapooa-Kikapú & Algonquina & & & 80 \\
\hline Migrantes & 50 grupos etnolingüísticos & 5.025 & 11.675 & Población fluctuante \\
\hline Total & & 58.773 & 95.507 & 137.560 \\
\hline \hline
\end{tabular}

* HLI: Hablantes de Lengua Indígena. ** HPI: Hogares con Población Indígena. *** Conteos comunitarios, información de autoridades tradicionales, por autoadscripción y reconocimiento comunitario.

Fuente: modificado de Diana Luque, Angelina Martínez-Yrízar, Alberto Búrquez, Eduwiges Gómez, Alejandro Nava \& Moisés Rivera (2012b)

Las condiciones de vida en las áreas rurales e indígenas en Sonora limitan los alcances de la educación de los hijos, debido a que los padres pasan largas jornadas en el campo, en trabajos poco redituables. Como consecuencia, el desarrollo de los niños presenta deficiencias en la ejecución de sus habilidades (Vera, 2006). La estimulación que proporcionan los padres a los niños se relaciona con la historia de socialización de las madres, percepción de la crianza, educación, nivel económico y el contexto cultural con normas de comportamiento social establecidas y transmitidas de generación en generación. El control de la conducta infantil y los procesos de salud y nutrición resultan prácticas concretas de crianza en la familia (Peña-Ramos, Aguilar-Rodríguez \& Vera-Noriega, 2005). Es decir, el diseño 
de la política pública educativa no ha sido capaz de integrar la complejidad de la diversidad cultural de México.

Un estudio cuantitativo que comparó hogares con o sin programa no escolarizado de educación inicial, en relación con los niveles de estrés de la madre y las condiciones del cuidado del niño, encontró que el patrón de comportamiento que siguen las madres de las comunidades rurales e indígenas del sur del Sonora en condiciones de pobreza extrema se distinguen por su desamparo, desesperanza y sobre todo, percepción de altos niveles de riesgo que les hacen asumir la vida con un sentido desdeñoso, sin sorpresas, esperanzas o excedentes, pues hay poco qué contar y mucho qué hacer para lograr el recurso necesario para el alimento diario.

Igualmente, el niño se desarrolla con una visión colectivista, solidaria y respetuosa de las prácticas culturales, bajo un paradigma autóctono de libertad que se caracteriza por la oportunidad de aprender de los pares y hermanos. Así, mientras el niño juega y se relaciona con otros, los padres monitorean el riesgo que ello conlleva (Vera \& Rodríguez, 2011). Cada comunidad manifiesta distintos procesos de transformación cultural en su relación con la sociedad nacional, lo cual redunda directamente en una percepción diferenciada sobre la utilidad de la educación.

Por otro lado, la percepción de la escuela cambia sustantivamente cuando la familia tiene un sueldo fijo. La concepción de la educación se modifica, porque el salario fijo y predecible permite establecer planes y estructurar el futuro, a diferencia de las familias que trabajan por jornal para los hacendados o terratenientes, y no saben si al día siguiente serán contratadas. Estas últimas familias perciben la escuela como un sistema de socialización o de cuidado de los infantes, más que como una inversión a largo plazo. Los datos del informe destacan que en los pueblos hay envidia, resentimiento, falta de solidaridad y de confianza, lo cual establece condiciones que obstaculizan el desarrollo del programa de educación inicial (Vera, 2010).

Los grupos indígenas presentan una transformación demográfica, económica, educativa y de organización social, debido a las exigencias que les plantea la modernidad, que se exacerba por su problemática estructural de marginación histórica y despojo territorial. Por ello, se requiere desarrollar nuevos métodos y técnicas para generar teorías que sean capaces de evaluar y sustentar características oportunas enmarcadas por las especificidades culturales e individuales de carácter dinámico y cotidiano del contexto (Organización Mundial de la Salud, OMS, 2004).

La calidad de la relación de pareja y el trato positivo influyen de manera directa en la educación de los hijos; las evidencias demuestran la importancia de la relación de pareja para comprender la percepción de la madre en el cuidado de los hijos, como la estimulación, la atención, el apoyo emocional y afectivo en el desarrollo y educación del infante (PeñaRamos, Aguilar-Rodríguez \& Vera-Noriega, 2005). Por lo tanto, la comunicación en el hogar permite la riqueza de lenguaje en los niños, mediante las repeticiones, preguntas y demandas que realizan los padres. La familia también es conocida como un grupo de personas nucleadas por relaciones de parentesco (naturaleza biológica), alrededor de la forma monógama y heterosexual, que cumple funciones esenciales en los cuidados asistenciales, la estimulación del niño y, en consecuencia, el fortalecimiento en el desarrollo infantil. Jesús Francisco Laborín-Álvarez y José Ángel Vera-Noriega (1998) reportan que las madres desarrollan sensibilidad al interactuar con sus hijos, lo que les permite adaptar su propia conducta a las exigencias y demandas específicas del niño, estructurando la naturaleza de las experiencias de sus hijos en el hogar. 


\section{Condiciones escolares de alumnos indígenas}

Martín Carnoy, Lucrecia Santibáñez, Alma Maldonado e Imanol Ordorika (2002) señalan que los profesionales indígenas empleados en el sector educativo ganan, en su mayoría, menos de tres salarios mínimos. Ellos enuncian cinco áreas claves que restringen el acceso a la educación superior: la distancia geográfica, las barreras culturales, las barreras económicas, la calidad educativa y de oportunidades, y los factores discriminatorios. Un patrón común de los casos de éxito está formado por la motivación y el empuje individual y/o por parte de familiares y maestros.

Las dificultades de adaptación a las disciplinas o hábitos que exigen los mercados laborales modernos es otro factor que incide en esa deserción. Según Carnoy, Santibáñez, Maldonado y Ordorika (2002), las poblaciones indígenas necesitan tener acceso a todas las carreras que se ofrecen dentro de la educación superior, no solo a las que los políticos piensan son apropiadas para ellos.

Respecto al Estado de Sonora, la mayor cobertura para la formación indígena (tabla 2), se limita al nivel básico pues las escuelas adscritas a la Dirección de Educación Indígena son en su mayoría preescolares, le siguen la telesecundaria y, por último, la primaria. En la mayoría de los casos, los profesores contratados no hablan la lengua del grupo a la que se adscribe el alumno o hablan una diferente.

Tabla 2

Escuelas indígenas del nivel básico en el Estado de Sonora, ciclo escolar 2008-2009

\begin{tabular}{l|c|c|c|c|c}
\hline & & \multicolumn{2}{|c}{ Docentes } & \multicolumn{2}{c}{ Alumnos } \\
\hline \multicolumn{1}{c|}{ Modalidades } & Número de escuelas & Hombres & Mujeres & Hombres & Mujeres \\
\hline Preescolares indígenas & $227^{\mathrm{a}}$ & 7 & 363 & 3.580 & 3.472 \\
\hline Preescolar comunitariab & 358 & 93 & 244 & 1.784 & 1.339 \\
\hline Primarias indígenas & $110^{c}$ & 198 & 111 & 3.070 & 2.971 \\
\hline Primarias comunitarias & 90 & 49 & 45 & 310 & 263 \\
\hline Telesecundaria & $294^{\mathrm{d}}$ & 525 & 307 & 7.400 & 6.871 \\
\hline \hline
\end{tabular}

a De estas escuelas, 100 son unitarias de organización completa, es decir, un solo maestro atiende a los tres grados.

b La educación comunitaria es una de las respuestas del Ejecutivo Federal para cumplir el mandato constitucional de ofrecer educación básica a la población que, por diversas circunstancias, no tiene acceso en condiciones de equidad a la oferta del sistema educativo regular. Una de las características principales de este modelo es su método de trabajo multigrado, que propicia el trabajo conjunto de alumnos con diferentes conocimientos y edades.

c De estas escuelas, 85 son de organización multigrado, que pueden ser unitarias, bidocentes y tridocentes.

d De estas escuelas, 107 son unitarias y 57 bidocentes.

Fuente: elaborado con base en las ESTADÍSTICAS continuas del formato 911 (inicio del ciclo escolar 2008/2009), Dirección General de Planeación y Programación, de la Secretaría de Educación Pública, DGPP-SEP, y del Instituto Nacional para la Evaluación de la Educación, INEE (2009).

Por otra parte, el hecho de que se haya incrementado el número de escuelas que ofrecen educación a la población indígena no necesariamente se traduce en mejor desempeño académico para los estudiantes indígenas. En este sentido, el Instituto de Innovación y Evaluación Educativa del Estado 
de Sonora, IIEEES (2011), reporta que los estudiantes de las modalidades indígenas son los que obtienen puntajes por debajo de la media en la evaluación estatal del desempeño escolar en primaria y secundaria (ver tabla 3).

Tabla 3

Resultados obtenidos por las escuelas indígenas frente al promedio estatal en la evaluación del desempeño escolar en primaria y secundaria, 2011

\begin{tabular}{c|c|c|c|c|c|c|c|c}
\hline \multicolumn{4}{c|}{ Promedio primarias indígenas } & \multicolumn{4}{c}{ Promedio primarias totales } \\
\hline Número de escuelas & Español & Matemáticas & Geografía & Número de escuelas & Español & Matemáticas & Geografía \\
\hline 90 & 501 & 503 & 495 & 1.678 & 559 & 564 & 524 \\
\hline \multicolumn{4}{c|}{ Promedio telesecundaria } & \multicolumn{3}{c|}{ Promedio secundarias totales } \\
\hline Número de escuelas & Español & Matemáticas & Geografía & Número de escuelas & Español & Matemáticas & Geografía \\
\hline 268 & 491 & 553 & 516 & 634 & 697 & 543 & 519 \\
\hline
\end{tabular}

Fuente: elaborado con datos del Instituto de Innovación y Evaluación Educativa del Estado de Sonora, IIEEES, 2011

\section{Objetivo}

Analizar la infraestructura y calidad educativa para la formación de los descendientes de los siete pueblos indígenas originarios de Sonora y establecer diferencias entre los siete grupos indígenas del Estado de Sonora.

\section{Metodología}

\section{Participantes}

Participaron 1.329 personas, 928 mujeres y 401 hombres, tienen un promedio de 50 años de edad y una desviación estándar de 16 años y pertenecen a alguno de los siete pueblos originarios de Sonora — cucapá, seri, yaqui, mayo, pápago, pima y guarijío-. A partir de las estadísticas del INEGI 2005, en los grupos con mayor número de sujetos, se realizó un muestreo en las localidades de mayor población en sus territorios, con resolución presidencial (núcleos agrarios) en el caso mayo, y con reconocimiento oficial, en el caso yaqui. Mientras que en los otros pueblos se aplicó una encuesta en cada vivienda. La muestra se distribuye como sigue: cucapás, 34; guarijíos, 128; mayos, 419; pápagos, 135; pimas, 126; seris, 81 y yaquis, 406 .

Las localidades se encuentran en 16 municipios del Estado y son: (cucapás) Pozas de Arvizu y San Luis Río Colorado; (guarijíos) Bavícora, Burapaco, Colonia Makorahui, Mesa Colorada y Mochibampo; (mayos) Bacabachi, Bachantahui, Bayajorit, Buaysiacobe, Camahuiroa, El Caro, El Júpare, El Sahuaral, Etchohuaquila, La Primavera, La Vasconia, Las Bocas, Loma Etchoropo, Los Pollos, Masiaca, Mochipaco II, Moroncarit, Óraba, Yavaritos; (pápagos) Caborca, Pitiquito, Puerto Peñasco, Quitovac y Sonoyta; (pimas) El Kipor, La Dura, Maycoba, San Juan Diego y rancherías del núcleo agrario Maycoba; (yaquis) Bahía de Lobos, Belem, Huirivis, Lomas de Bácum, Lomas de Guamúchil, Pótam, Rahum, Torim, Vícam pueblo; (seris) Punta Chueca. Finalmente, las personas tenían un promedio de 42 años y desviación estándar de 19 años viviendo en el lugar donde se encontraban cuando se les aplicó la encuesta. 


\section{Instrumento}

La encuesta de Biodiversidad y Subsistencia ${ }^{5}$ que se les aplicó es una herramienta semiestructurada que consta de 47 preguntas e indaga sobre diversos temas, como educación, alimentación, salud, vivienda y recursos naturales. Además de datos de identificación, como edad, sexo, grupo étnico y antigüedad en la comunidad. En el rubro de educación, fueron documentados tres reactivos (escolaridad, sabe leer, sabe escribir) y cuatro fueron interpretativos, porque están vinculadas a las creencias y las percepciones de las personas (tabla 4).

Tabla 4

Características de la encuesta de subsistencia y biodiversidad

\begin{tabular}{l|c|l|l}
\hline \multicolumn{1}{c|}{ Dimensiones } & Número de reactivos & \multicolumn{1}{c}{ Tipo de reactivos } & \multicolumn{1}{c}{ Ejemplo de reactivos } \\
\hline Educación & 7 & Dicotómicos y politómicos & $\begin{array}{l}\text { ¿Faltan planteles educativos para que } \\
\text { los hijos sigan estudiando? }\end{array}$ \\
\hline Alimentación & 3 & Abiertas y politómicos & ¿Qué comió ayer? \\
\hline Vivienda & 7 & Dicotómicos y politómicos & ¿Su vivienda está hecha de...? \\
\hline Salud & 8 & Abiertas y dicotómicas & ¿Falta un centro de salud? \\
\hline Empleo & 8 & Dicotómicos y politómicos & ¿Cuál es su ocupación? \\
\hline Recursos Naturales & 5 & $\begin{array}{l}\text { Abiertas, dicotómicos } \\
\text { y politómicos }\end{array}$ & $\begin{array}{l}\text { ¿En la zona observa disminución y/o } \\
\text { desaparición de flora y fauna? }\end{array}$ \\
\hline \hline
\end{tabular}

Fuente: elaboración propia

\section{Procedimiento}

La aplicación de la Encuesta de subsistencia y biodiversidad se efectuó durante el periodo de abril de 2010 a mayo de 2011. Para ello, previamente se establecieron contactos con las autoridades correspondientes (tradicionales y funcionarios), para informar sobre los objetivos del proyecto. Posteriormente, se formaron grupos de 5 a 15 integrantes con personal del Centro de Investigación en Alimentación y Desarrollo, CIAD, Asociación Civil y jóvenes pertenecientes a la misma etnia que se incorporaron como encuestadores interculturales. En este sentido, se realizó una capacitación de dos horas con la finalidad de que los jóvenes conocieran la estructura de la entrevista, la forma de plantear las preguntas, el llenado de la encuesta y la edad de la persona a entrevistar.

Igualmente, la aplicación de la encuesta fue de manera individual, a la persona de mayor edad que estaba en el momento de la aplicación, con visitas domiciliarias y en algunos casos las entrevistas se realizaron en el idioma de la etnia. Cabe señalar que los jóvenes que se integraron como encuestadores recibieron estímulos económicos. Los datos se capturaron en el programa de Excel 2007 y se analizaron en SPSS 18.

\section{Resultados}

Los resultados de la Encuesta de subsistencia y biodiversidad aplicada en 49 localidades, en la que participaron 1.329 sujetos que pertenecen a alguno de los siete grupos indígenas que tienen núcleos agrarios

5 La encuesta Subsistencia y Biodiversidad es uno de los instrumentos de investigación utilizados en el proyecto EDESPIS, Capítulo Territorio y Recursos Naturales (Luque, Martínez-Yrízar, Búrquez, Gómez, Nava \& Rivera, 2012a). 
legalmente constituidos en el Estado de Sonora, demostró que el 79,5\% de los sujetos saben leer y 77,5\% saben escribir.

La mayoría de los sujetos tiene un nivel de escolaridad básica, no obstante, el 23,8\% nunca asistió a la escuela. Por otro lado, cuando se les hacen preguntas relacionadas con la infraestructura para la formación educativa, el $69,7 \%$ de ellos dijo que hacen falta planteles educativos que pueden ser desde primarias $(27,7 \%)$, secundarias $(14,7 \%)$, preparatorias (32,3\%), y universidades (11,7\%). Asimismo, el 58,8\% expresó la falta de maestros más capacitados en los planteles, el 78,2\% señaló faltas de recursos económicos para que los niños y jóvenes puedan continuar en la escuela y el $48,2 \%$ dijo que los niños no cuentan con útiles escolares (tabla 5).

Tabla 5

Estado actual de educación en pueblos indígenas de Sonora, derivado de la encuesta

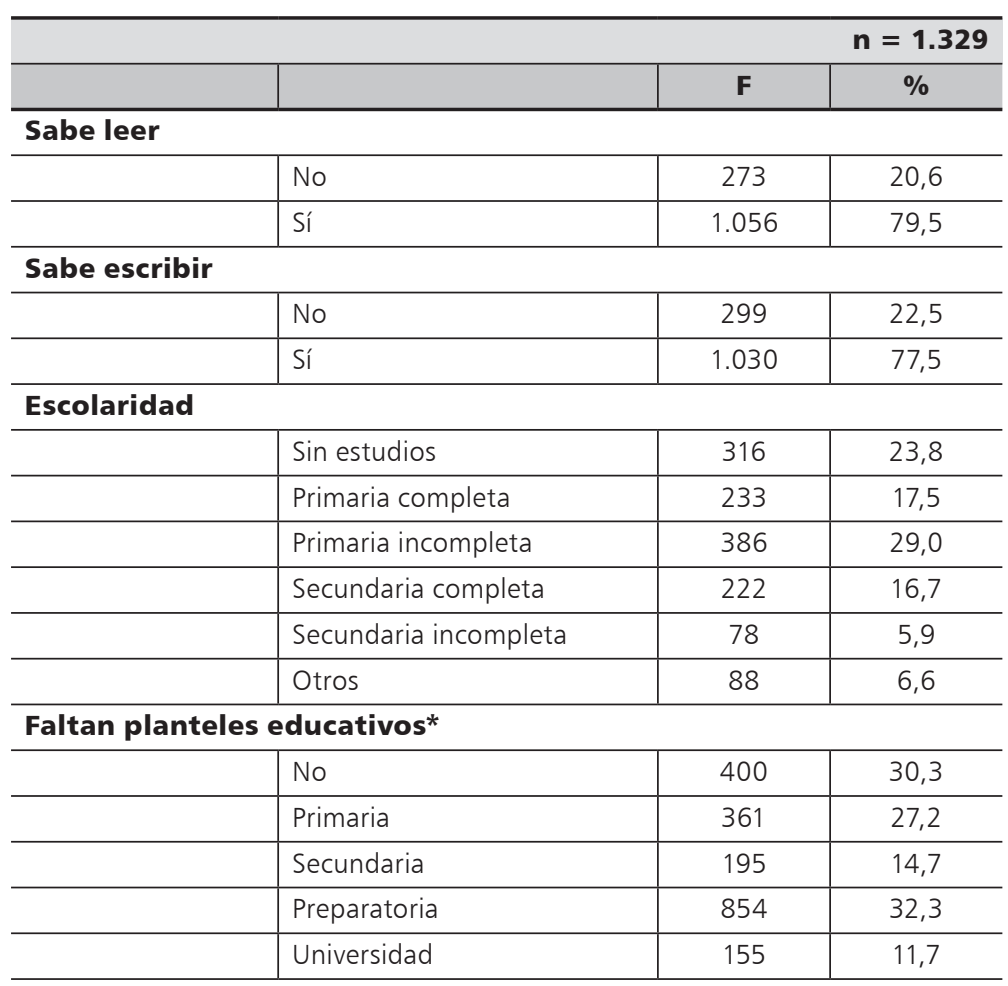

Faltan maestros capacitados

\begin{tabular}{l|l|c|c}
\hline & Sin información & 92 & 6,9 \\
\hline & No & 456 & 34,3 \\
\hline & Sí & 781 & 58,8 \\
\hline
\end{tabular}

Faltan recursos para seguir estudiando

\begin{tabular}{c|l|c|c}
\hline & Sin información & 89 & 6,7 \\
\hline & No & 201 & 15,1 \\
\hline & Sí & 1.039 & 78,2 \\
\hline
\end{tabular}

Los niños cuentan con útiles escolares

\begin{tabular}{c|l|c|c}
\hline & Sin información & 91 & 6,8 \\
\hline & No & 640 & 48,2 \\
\hline & Sí & 598 & 45,0 \\
\hline \hline
\end{tabular}

* Los porcentajes no suman 100\%, porque la persona podía elegir más de una opción.

Fuente: elaboración propia con datos obtenidos de la encuesta de Subsistencia y Biodiversidad 2011. 
Por otro lado, el análisis entre los grupos étnicos vinculado con la interpretación que tienen sobre la infraestructura educativa para la formación de sus hijos, denota diferencias importantes. Así, el 85,7\% de los pimas dijo que en su comunidad hacen falta escuelas primarias; por el contrario, solo el $17,6 \%$ de los cucapás percibe esta falta. El grupo que más demanda secundaria es el mayo y el cucapá es el que menos la demanda. Para la demanda del nivel medio superior, la percepción es más homogénea entre los grupos, aunque los guarijíos $(51,6 \%)$ encabezan la lista, le siguen yaquis $(42,9 \%)$ y mayos $(34,4 \%)$, el último, los cucapás. Los pápagos figuran por primera vez en la percepción de falta de universidades en su contexto (tabla 6).

Tabla 6

Estado actual de educación por grupo étnico

\begin{tabular}{|c|c|c|c|c|c|c|c|}
\hline \multicolumn{8}{|c|}{ Faltan recursos económicos para que los niños sigan en la escuela } \\
\hline No & 20,6 & 11,7 & 14,3 & 37,0 & 12,7 & --- & 13,1 \\
\hline Sí & 79,4 & 82,0 & 85,7 & 63,0 & 87,3 & --- & 86,9 \\
\hline \multicolumn{8}{|c|}{ Los niños cuentan con útiles escolares } \\
\hline No & 55,9 & 37,5 & 41,5 & 65,2 & 58,7 & --- & 58,4 \\
\hline Sí & 44,1 & 55,5 & 58,5 & 34,8 & 40,5 & --- & 41,6 \\
\hline
\end{tabular}

* Los porcentajes no suman 100\%, porque la persona encuestada podía elegir más de una opción.

Fuente: elaboración propia con datos obtenidos de la encuesta de Subsistencia y Biodiversidad 2011

\section{Percepción del contexto social y educativo}

Con el objetivo de verificar si el factor grupo étnico establece diferencias significativas se llevó a cabo un análisis con la prueba Chi-Cuadrado, los resultados indican que para cada uno de las variables hay diferencias estadísticas con respecto al grupo indígena al que los encuestados pertenecen (tabla 7). Con relación a si saben leer, las diferencias son estadísticamente significativas $\left(X^{2}=194,448 ; P \leq 0,01\right)$. Si se analiza por grupo étnico, los guarijíos son los que tienen las tasas más altas de analfabetismo (de cada 100 guarijíos entrevistados solo 48 saben leer), seguido por los pimas pues de cada 100 pimas encuestados 52 saben leer, mientras que los que tienen menor tasa son los cucapás (todos los cucapás encuestados saben 
leer) y los pápagos (98 de cada 100 sabe leer). Sin embargo, si se analiza entre los grupos, de cada 100 entrevistados que saben leer 35 son mayos; 30, yaquis; 13 , pápagos; 6 , pimas; 6 , guarijíos; 7 , seris; 3 , cucapás.

Tabla 7

Análisis prueba ji-cuadrada para el contexto social y educativo, a partir de la base de datos de la encuesta Subsistencia y Biodiversidad

\begin{tabular}{l|c|c|c}
\hline Variables & \multicolumn{1}{c}{$\boldsymbol{X}^{\mathbf{2}}$} & Phi & $\begin{array}{c}\text { Coeficiente } \\
\text { de contingencia }\end{array}$ \\
\hline Sabe leer & $194,448^{* *}$ &, $383^{* *}$ &, $357^{* *}$ \\
\hline Sabe escribir & $174,546^{* *}$ &, $362^{* *}$ &, $341^{* *}$ \\
\hline $\begin{array}{l}\text { En su familia hablan } \\
\text { en lengua materna }\end{array}$ & $370,517^{* *}$ &, $529^{* *}$ &, $468^{* *}$ \\
\hline La vivienda es propia & $23,961^{* *}$ &, $140^{* *}$ &, $139^{* *}$ \\
\hline Faltan maestros capacitados & $86,957^{* *}$ &, $265^{* *}$ &, $256^{* *}$ \\
\hline $\begin{array}{l}\text { Faltan recursos para seguir } \\
\text { los estudios }\end{array}$ & $50,033^{* *}$ &, $201^{* *}$ &, $197^{* *}$ \\
\hline Cuenta con útiles escolares & $43,920^{* *}$ &, $188^{* *}$ &, $185^{* *}$ \\
\hline \hline
\end{tabular}

En relación con la pregunta sobre si saben escribir, se encontró que los que tienen más probabilidades de saber escribir son los pápagos (98 de cada 100 pápagos saben escribir), seguido por los cucapás (solo 1 de los 34 cucapás encuestados no sabe escribir), contrario a los guarijíos que tienen menos probabilidades pues 48 de cada 100 saben escribir, le siguen los pimas (52 de cada 100 saben leer). Por otra parte, si se analiza entre los grupos, de cada 100 entrevistados que saben escribir 35 son mayos; 30, yaquis; 13 , pápagos; 6, pimas; 6, guarijíos; 7, seris y 3, cucapás. Las comunidades pápago y cucapá presentan los porcentajes más altos de población urbana, es decir, ya abandonó su territorio rural, mientras que los guarijíos viven en comunidades rurales en un $100 \%$.

Referente a la práctica de la lengua materna en la familia, 99 de cada 100 seris hablan frecuentemente en su lengua, les siguen los guarijíos con una proporción es 91 de cada 100. Por el contrario, los pápagos (1 de cada 100 pápagos hablan en su lengua frecuentemente) y los cucapás (24 de cada 100 cucapás hablan en su lengua frecuentemente) son los que la practican con menor frecuencia. Por otro lado, si se analiza entre los grupos, de cada 100 entrevistados que hablan en su lengua frecuentemente: 38 son yaquis; 25 , mayos; 14 , guarijíos; 11 , pimas; 10 , seris; 1 , pápago y 1, cucapá.

Por otro lado, el 91\% de los encuestados vive en casa propia: Ios seris (99 de cada 100 viven en casa propia) y los yaquis (93 de cada 100 viven en casa propia) presentan las tasas más altas y los pápagos (82 de cada 100 viven en casa propia) y los guarijíos (84 de cada 100 viven en casa propia) las tasas más bajas. Un análisis entre los grupos da cuenta que de cada 100 sujetos con vivienda propia: 2 son cucapás; 9, guarijíos; 32, mayos; 8 , pápagos; 10, pimas; 7, seris y 32, yaquis. A pesar de que la casa es propia, las condiciones de la vivienda generalmente son muy precarias y con pocas instalaciones sanitarias adecuadas. Esto está muy generalizado en todos los grupos, excepto aquellos que ya están en las ciudades.

Con relación a la calidad de la educación, vinculado a la exigencia de maestros mejor capacitados en las escuelas, 84 de cada 100 guarijíos y 79 de cada 100 pimas consideran que hacen falta maestros mejor capacitados; 
mientras que de los cucapás, solo el 23,5\% de los encuestados dijo que hacen falta maestros mejor capacitados y de los mayos, 53 de cada 100 contestaron que hacen falta maestros mejor capacitados. En este sentido, de cada 100 sujetos que afirman de manera positiva la falta de maestros mejor capacitados, 1 es cucapá; 13 son guarijíos; 28, mayos; 9, pápagos; 13, pimas; y 36, yaquis. Para este análisis, no se considera a los seris, pero en la observación participativa han manifestado la necesidad de maestros mejor capacitados, aunque esto signifique que la educación no sea en la lengua materna.

De la misma manera, 87 de cada 100 guarijíos, y 87 de cada 100 pimas contestaron que hacen falta recursos económicos para que los jóvenes puedan continuar con sus estudios, mientras que los pápagos (63 de cada 100) y cucapás (el 79,4\% de los cucapás encuestados dijo que hacen falta recursos) presentan las tasas relativamente más bajas. Así, de cada 100 sujetos que afirman de manera positiva la falta de recursos para que los jóvenes continúen estudiando: 3 son cucapás; 10, guarijíos; 35, mayos; 8, pápagos; 11, pimas, y 34, yaquis. Es decir, la falta de recursos económicos para continuar con los estudios es una problemática muy generalizada en los grupos indígenas de Sonora. Para este análisis no se consideró a los seris.

Por último, 59 de cada 100 guarijíos y 58 de cada 100 mayos registran las tasas más altas de los que dijeron que los jóvenes reciben apoyo en útiles escolares, mientras que los pápagos (35 de cada 100 pápagos) y pimas (41 de cada 100 pimas) registran las tasas más bajas. De cada 100 sujetos que afirman que los niños reciben útiles escolares: 3 son cucapás; 12, guarijíos; 41, mayos; 7, pápagos; 9, pimas, y 28, yaquis. Para este análisis no se consideró a los seris.

\section{Discusión}

Tal como señalan algunas investigaciones previas (Castillo, 2005; Muñoz, 2001; Schmelkes, 2013a), pese a los avances para generar estructuras y oportunidades educativas entre las comunidades indígenas, estos apenas se limitan a la educación básica. En el contexto de los siete pueblos originarios del Estado de Sonora, prevalece una percepción de falta de infraestructura educativa para la formación de los niños, como escuelas y docentes bien capacitados, apoyos para la compra de útiles escolares $y$, por ende, de un nivel de escolaridad básica. En un contexto en que la economía indígena no es suficiente siquiera para cubrir gastos de alimentación mucho menos para cubrir gastos para la educación ${ }^{6}$, es necesario tener buenas escuelas y

6 Hilda Jabalí, regidora étnica de Álamos, Mesa Colorada, municipio Álamos, Sonora, 2011. maestros preparados que se adapten a las necesidades de sus estudiantes, pero también se necesita promover programas que sean capaces de amortiguar los gastos de la familia, para mejorar los resultados en la formación indígena en la búsqueda por alcanzar su pleno derecho a la educación.

Por otro lado, en los resultados se puede dibujar que las condiciones educativas son heterogéneos entre los pueblos indígenas. Estas diferencias son en parte, consistentes con lo que ha señalado Federico Navarrete-Linares (2008, p. 108) como: "la clara desventaja de los indígenas en el terreno educativo se debe, en primer lugar, a la tradicional desatención por parte del gobierno: por hablar una lengua diferente y por vivir en las regiones más remotas del país (por ejemplo, los guarijíos y pimas en Sonora), las comunidades indígenas no han recibido los mismos servicios educativos que otros grupos y comunidades. El pequeño tamaño de muchos asentamientos indígenas también hace muy costoso y poco eficiente proporcionarles servicios de salud, vivienda y educativos; por ello, en la mayoría de los poblados, las escuelas son demasiado pequeñas para tener todos los grados".

Sin embargo, a partir del presente estudio, se puede afirmar que las diferencias en las condiciones educativas pueden tener otros orígenes además del de la lejanía, o sumarse a este, pues por ejemplo, en el caso de los pápagos, su relativo mayor grado de alfabetización está más relacionado con su emigración obligada a las ciudades, debido a la pérdida de su territorio y al acceso a recursos naturales como el agua (que los despojó de su medio de subsistencia tradicional).

Los pápagos cuentan con el apoyo económico de sus pares que habitan en Estados Unidos en Arizona que tienen mejor nivel económico que los mexicanos; parte de este apoyo se traduce en becas para estudios universitarios. El único núcleo agrario pápago que continua habitado es Quitovac, en donde se ubicó una escuela rural que atendía a las rancherías aledañas, lo cual benefició a este grupo de pápagos y les dio un medio de subsistencia al ser contratados para su atención.

Así se puede plantear una hipótesis que relacione el acceso a los recursos naturales de la subsistencia tradicional, es decir, que aún encuentren alimento en su territorio originario, lo cual incide en el grado de intercambio monetario para subsistir, con el sentido que le puedan dar a esforzarse para tener acceso a una educación que les prometa una mayor inserción en los mercados laborales asalariados.

Además, el uso de la lengua se relaciona con el nivel de alfabetización, pues en las comunidades señalan que por ir a la escuela se pierden las tradiciones, es decir, la identidad cultural comunitaria que sirve para saber, transmitir y comprender el patrimonio cultural 
de los pueblos. Se requiere reparar este daño con otro tipo de educación, que en lugar de debilitar el uso de la lengua, la fortalezca (Blanco, 2006).

La interculturalidad como una política educativa del Estado mexicano debe reconocer que los pueblos originarios son herederos de una cultura "diferente" en el sentido profundo de la cosmovisión, con lengua, historia y sentido comunitario propio y que aún aspiran a una autonomía política y autogestión del territorio, para superar el proceso de colonización europea, las políticas de exterminio y el despojo territorial, que van en total detrimento de estos pueblos y generan una inequidad política y económica (CDI-PNUD, 2010).

\section{Conclusiones}

Las condiciones educativas de la población indígena en México también son evidentes en los pueblos originarios del Estado de Sonora. Estas condiciones educativas se refieren tanto al grado de escolaridad como a la infraestructura, calidad de los maestros y apoyos educativos. La atención a la problemática educativa a este sector de la población, con un enfoque intercultural e inclusivo enfrenta dilemas que se relacionan con las diferencias culturales-lingüísticas, con la marginación política y económica estructural e histórica.

Además, este proceso en los pueblos originarios de Sonora es heterogéneo, pues cada pueblo presenta su propia problemática o grado de transformación cultural. Esto es, algunos grupos continúan fincando la identidad comunitaria en la subsistencia tradicional como base de la autonomía política, mientras otros grupos pugnan por tener acceso a la educación de calidad de "los blancos", aun cuando el fantasma del desempleo esté haciendo estragos en muchos casos. Así, la problemática educativa de las comunidades indígenas va más allá de la inversión de recursos económicos en infraestructura y en becas escolares.

Las limitaciones del estudio se derivan de que en esta ocasión se registró la "percepción" que tienen los adultos entrevistados sobre la problemática educativa, y aún falta contrastarla con las percepciones de las generaciones jóvenes, funcionarios y actores sociales estratégicos involucrados en esta temática. También falta exponer la heterogeneidad en cuanto a las perspectivas de desarrollo y por ende, del tipo de educación requerida en todas estas comunidades, pues en general, tienen casi nula intervención en este tema. En este sentido, es de mayor importancia integrar como marco de referencia las demandas contemporáneas de los pueblos indígenas, expresadas tanto en el ámbito local, como en el seno de las Naciones Unidas, entre las que destacan el derecho a la libre autodeterminación, a la autogestión de su desarrollo, con el territorio y el legado biocultural comunitario como asuntos centrales de sus agendas como pueblos.

Además de las investigaciones sobre los procesos de adquisición y desarrollo de procesos lógico-matemáticos y lectoescritura de su lengua materna y la del español, también habría que indagar las expectativas del joven y los propósitos de la comunidad como fundamento intercultural que garantice la inclusión y que sea capaz de adaptarse no solo a la diversidad cultural y lingüística, sino a la diversidad de aspiraciones que caracteriza a hombres y mujeres y generaciones jóvenes de los distintos pueblos originarios de Sonora (Blanco, 2006; Schmelkes, 2013b). Y con ello, lograr el establecimiento progresivo de relaciones dialógicas y proyectos comunes (Almeida-Acosta, 2013).

\section{Agradecimientos}

A las autoridades tradicionales de cada comunidad, y a los jóvenes que se integraron como "encuestadores interculturales". Se agradece también la colaboración en campo de la Comisión Nacional para el Desarrollo de los Pueblos Indígenas (CDI), el Consejo Nacional para la Cultura y las Artes (CONACULTA) y el Instituto Nacional de Antropología e Historia (INAH) y, por supuesto, al Centro de Investigación en Alimentación y Desarrollo, CIAD.

\section{Sobre los autores}

José Ángel Vera-Noriega hace parte del Sistema Nacional de Investigadores Nivel II. Investigador Nacional Nivel I C, Consejo Nacional de Investigación de Brasil. Investigador Titular D. Profesor de la maestría en desarrollo regional y del doctorado en ciencias. Líneas de investigación: estilos de crianza, evaluación de programas en educación y salud y etnopsicología y psicología transcultural.

Diana Luque-Agraz es investigadora titular. Profesora de la maestría en desarrollo regional y del doctorado en ciencias, especialidad en desarrollo regional. SNI-1 (2010). Línea de investigación: gestión ambiental del desarrollo.

Gildardo Bautista-Hernández es estudiante de posgrado en desarrollo regional. Apoyo técnico en el proyecto Estado de Desarrollo de los Pueblos Indígenas de Sonora, EDESPIS, Capítulo Territorio y recursos naturales.

\section{Referencias}

Almeida-Acosta, Eduardo (septiembre, 2013). La dignidad de los pueblos originarios. En Katherine label Herazo-González (dir.). Memorias del $1^{\text {er }}$ Coloquio Internacional de Psicología Social Comunitaria en Nuestra América. Una mirada a los pueblos originarios, 15-31. México: Universidad Nacional Autónoma de México, UNAM. 
Avilés, K. (2011). De cada 100 indígenas 2 llegan a la educación superior: expertos. Periódico La Jornada, 42 (10 de agosto de 2011). Disponible en: http://www.jornada.unam.mx/2011/08/10/sociedad/042n1soc

Baronnet, B. (2011). La apuesta de las escuelas zapatistas de Chiapas por descolonizar la educación en los pueblos campesinos mayas. Decisio, Saberes para la Acción en Educación de Adultos, 30, 3943. Disponible en: http://tumbi.crefal.edu.mx/decisio/images/pdf/ decisio_30/decisio30_saber7.pdf

Blanco, R. (2006). La equidad y la inclusión social: uno de los desafíos de la educación y la escuela hoy. Revista Electrónica Iberoamericana sobre Calidad, Eficacia y Cambio en Educación, REICE, 4 (3), 1-15. Disponible en: http://www.rinace.net/arts/vol4num3/art1.pdf

Boege, E. (2008). El patrimonio biocultural de los pueblos indígenas de México. Hacia la conservación in situ de la biodiversidad y la agrodiversidad de los territorios indígenas. México: Instituto Nacional de Antropología e Historia, INAH, Consejo Nacional para la Cultura y las Artes, Conaculta, Comisión Nacional para el Desarrollo de los Pueblos Indígenas, CDI. Disponible en: http://www.cdi.gob.mx/index. php?option $=$ com_content\&task=view\&id $=937$

Carnoy, M.; Santibáñez, L.; Maldonado, A. \& Ordorika, I. (2002). Barreras de entrada a la educación superior y oportunidades profesionales para la población indígena mexicana. Revista Latinoamericana de Estudios Educativos, 32 (3), 9-43. Disponible en: http://www.redalyc. org/articulo.oa?id=27032302

Castillo, S. (2005). Programa de Apoyo a Estudiantes Indígenas en Instituciones de Educación Superior, PAEIIES: una alternativa institucional para el acceso, equidad, cobertura y calidad. México: Asociación Nacional de Universidades e Instituciones de Educación Superior, ANUIES.

Comisión Nacional para el Desarrollo de los Pueblos Indígenas, Programa de las Naciones Unidas para el Desarrollo, CDI-PNUD (2006). Informe sobre Desarrollo Humano de los Pueblos Indígenas de México 2006. México: Comisión Nacional para el Desarrollo de los Pueblos Indígenas, Programa de las Naciones Unidas para el Desarrollo, CDI-PNUD. Disponible en: http://www.cdi.gob.mx/idh/informe desarrollo_humano_pueblos_indigenas_mexico_2006.pdf

Comisión Nacional para el Desarrollo de los Pueblos Indígenas, Programa de las Naciones Unidas para el Desarrollo, CDI-PNUD (2010). Informe sobre Desarrollo Humano de los pueblos indígenas en México. El reto de la desigualdad de oportunidades. México: Comisión Nacional para el Desarrollo de los Pueblos Indígenas, Programa de las Naciones Unidas para el Desarrollo, CDI-PNUD. Disponible en: http:// www.cdi.gob.mx/index.php?option $=c o m \_d o c m a n \& t a s k=d o c$ details\&gid $=161 \&$ Itemid $=200019$

Comisión Nacional para el Desarrollo de los Pueblos Indígenas, CDI (2009). Índice de Reemplazo Etnolingüístico (IRE) 2000-2005. México: Comisión Nacional para el Desarrollo de los Pueblos Indígenas, CDI. Disponible en: http://www.cdi.gob.mx/index.php?option=com_cont ent\&task= view\&id $=1075 \&$ Itemid $=54$

Comisión Nacional para el Desarrollo de los Pueblos Indígenas, CDI (2014). Programa Especial de los Pueblos Indígenas 2014-2018. México: Comisión Nacional para el Desarrollo de los Pueblos Indígenas, CDI. Disponible en: http://www.cdi.gob.mx/index.php?option=com content\&id $=3233$ 
Consejo Nacional de Población, CONAPO (2010). Encuesta Nacional de la Dinámica Demográfica (ENADID) 2009. México: Consejo Nacional de Población, CONAPO. Disponible en: http://conapo.gob.mx/ es/CONAPO/Encuesta_Nacional_de_la_Dinamica_Demografica_ ENADID

Consejo Nacional de Población, CONAPO (2012). De la población indígena de México 2000-2010. México: Consejo Nacional de Población, CONAPO. Disponible en: http://www.conapo.gob.mx/

Instituto de Innovación y Evaluación Educativa del Estado de Sonora, IIEEES (2011). Software Panorama Educativo de México 2011. Indicadores del Sistema Educativo Nacional. Educación básica. México: Instituto de Innovación y Evaluación Educativa del Estado de Sonora, IIEEES.

Instituto Nacional de Estadística y Geografía, INEGI (2005). /l Conteo de Población y Vivienda 2005. México: Instituto Nacional de Estadística y Geografía, INEGI. Disponible en: http://www.inegi.org.mx/est/ contenidos/proyectos/ccpv/cpv2005/

Instituto Nacional de Estadística y Geografía, INEGI (2010). Censo de Población y Vivienda. México: Instituto Nacional de Estadística y Geografía, INEGI. Disponible en: http://www3.inegi.org.mx/rnm/ index.php/catalog/71

Instituto Nacional para la Evaluación de la Educación, INEE (2013). Breve panorama educativo de la población indígena. México: Instituto Nacional de Evaluación Educativa, INEE. Disponible en: http:// publicaciones.inee.edu.mx/buscadorPub/P3/B/101/P3B101.pdf

Instituto Nacional para la Evaluación de la Educación, INEE (2009). Panorama educativo de México. Indicadores del Sistema Educativo nacional. Educación Básica. México: Instituto Nacional para la Evaluación de la Educación, INEE. Disponible en: http://www.inee.edu.mx/ index.php/70-publicaciones/panorama-educativo-capitulos/421panorama-educativo-de-mexico-indicadores-del-sistema-educativonacional-2009

Laborín-Álvarez, J. F. \& Vera-Noriega, J. Á. (1998). Secuencia e intercalamiento de estrategias de enseñanza en la interacción madre-hijo. La Psicología Social en México, 7, 388-393.

Luque, D.; Martínez, A.; Búrquez, A.; Gómez, E.; Nava, A. \& Rivera M. (2012a) Diagnóstico socioambiental de los pueblos indígenas de Sonora. Reporte técnico, Centro de Investigación en Alimentación y Desarrollo, CIAD, Hermosillo, Sonora.

Luque, D.; Martínez, A.; Búrquez, A.; Gómez, E.; Nava, A. \& Rivera M. (2012b). Política ambiental y territorios indígenas de Sonora. Estudios Sociales, XX, 253-280. Disponible en: http://www.redalyc.org/ articulo.oa? id $=41724972012$

Luque, D.; Martínez, A.; Búrquez, A.; Gómez, E.; Nava, A. \& Rivera M. (2012c). Pueblos indígenas de Sonora: ¿el agua es de todos? Región y Sociedad, 3, 53-88. Disponible en: http://www.scielo.org.mx/scielo. php?pid=S1870-39252012000600003\&script=sci_arttext

México (1917). Constitución Política de los Estados Unidos Mexicanos. Texto vigente disponible en: http://www.diputados.gob.mx/LeyesBiblio/ htm/1.htm

Moctezuma-Zamarrón, J.L. (2001). De pascolas y venados: adaptación, cambio y persistencia de las lenguas indígenas yaqui y mayo frente al español. México: Siglo XXI Editores. 
Muñoz-Cruz, H. (2001). Trayectoria de la política de educación en México. En Héctor Muñoz-Cruz (coord.). De práctica y ficciones comunicativa y cognitiva en educación básica, 433-494. México: Universidad Pedagógica Nacional, UPN Oaxaca, Universidad Autónoma Metropolitana, UAM.

Nabhan, G. P. (2003) Singing the Turtles to Sea. The Comcáac (Seri) Art and Science of Reptiles. Berkeley, Los Angeles, California: University of California Press.

Navarrete-Linares, F. (2008). Los pueblos indígenas de México. Pueblos indígenas del México contemporáneo. México: Comisión Nacional para el Desarrollo de los Pueblos Indígenas, CDI. Disponible en: https:// www.academia.edu/958886/Los_pueblos_ind\%C3\%ADgenas_ de_M\%C3\%A9xico

Organización de Naciones Unidas, ONU (2007). Declaración de las Naciones Unidas sobre los Derechos de los Pueblos Indígenas, A/61/L.67. Disponible en: http://www.un.org/esa/socdev/unpfii/documents/ DRIPS_es.pdf

Organización Mundial de la Salud, OMS (2004). Informe sobre la salud en el mundo 2004. Cambiemos el rumbo de la historia. París: Organización Mundial de la Salud, OMS. Disponible en: http://www. who.int/whr/2004/es/

Peña-Ramos, M. O.; Aguilar-Rodríguez, C. \& Vera-Noriega, J. Á. (2005). Pareja, estimulación y desarrollo del infante en zona rural en pobreza extrema. Revista Mexicana de Investigación Educativa, 10 (25), 559-576. Disponible en: http://www.redalyc.org/articulo. oa?id=14002516

Schmelkes. S. (2010). Indígenas rurales, migrantes, urbanos: una educación equivocada, otra educación posible. Pensamiento Iberoamericano, 7 (2), 203-222. Disponible en: http://www.corteidh.or.cr/tablas/ r24864.pdf

Schmelkes, S. (2013a). Educación para un México intercultural. Sinéctica, 40, 1-12. Disponible en: http://www.scielo.org.mx/pdf/sine/n40/ n40a2.pdf

Schmelkes, S. (2013b). Educación y pueblos indígenas: problemas de medición. Realidad, Datos y Espacio. Revista Internacional de Estadística y Geografía, 4 (1), 1-13. Disponible en: http://www.inegi. org.mx/RDE/RDE_08/RDE_08_Art1.html

Secretaría de Educación Pública, SEP, Sistema Nacional de Información Estadística Educativa, SNIE (2011). Sistemas de consulta interactiva de estadísticas educativas. Ciclo escolar 2008-2009. Disponible en: http://www.snie.sep.gob.mx/estadisticas_educativas.html

Secretaría de Educación Pública, SEP, Dirección General de Planeación y Programación, DGPP (2011). Sistema educativo de los Estados Unidos Mexicanos. Principales cifras ciclo escolar 2010-2011. México: Secretaría de Educación Pública, SEP. Disponible en: http:// planeacion.sep.gob.mx/estadistica-e-indicadores/estadisticas-eindicadores

Toledo, Víctor \& Barrera-Bassols, Narciso (2008). La memoria biocultural. La importancia ecológica de las sabidurías tradicionales. Barcelona: Icaria Editorial.

Vera-Noriega, J. Á. (2006). Psicopedagogía para el maestro de educación básica. Hermosillo, Sonora, México: Impresos Sindicato Nacional de Trabajadores de la Educación, SNTE, Sección 54. 
Vera-Noriega, J. Á. (2010). Etnopsicología de los procesos de crianza en poblaciones vulnerables. Revista Conducta: Psicología Científica al Alcance de Todos, 8 (16), 21- 31. Disponible en: http://www. investigacionesenpsicologia.com.mx/pdf1/Revista_CONDUCTANo 16.pdf

Vera, J. \& Rodríguez, C. (2011). Desarrollo y educación inicial en hogares de alta vulnerabilidad en el Estado de Sonora. En Eloy Méndez \& Alejandro Covarrubias (eds.). Estudios sobre Sonora 2010: instituciones, procesos socioespaciales, simbólica e imaginario, 271-288. México: Editorial Mora-Cantúa. 